\title{
Separation of Flavonoids and Salt in Bean Cake Disposed from Soy Sauce Manufacturing Process
}

\author{
Hideki Yamamoto, Fumiko TAKEUCHI, \\ Chika NAGANO, Ayako SASAKI and Junji SHIBATA \\ Department of Chemical Engineering, Faculty of Engineering, \\ Kansai University, 3-3-35, Yamatecho, Suita-shi, \\ Osaka 564-8680, Japan
}

Keywords: Separation, Flavonoids, Daidzein, Salt, Extraction

\begin{abstract}
Soy sauce cakes contain about 5-10 wt \% salt; therefore, incineration results in corrosion of the furnace and environmental problems related to, for example, dioxin generation. However, amino and organic acids, and soybean isoflavone, as well as a number of other useful components remain in the soy sauce cakes. The amount of desalination with water was $590 \mathrm{mg} / 10 \mathrm{~g}$-feed, while that with methanol was about $70 \%$ of this value. Desalination could not be performed successfully with acetone or $\boldsymbol{n}$-hexane. The amounts of flavonoid in the form of daidzein and genistein extracted from $10 \mathrm{~g}$ of soy sauce cake were 3.4-7.2 and 7.2-11.0 mg, respectively, while extraction with water was minimal. Moreover, the amount of flavonoids extracted increased while the amount of desalination decreased with aqueous alcohol solutions. The dual cylinder solid-liquid extractor presented here for separation of flavonoids and salt from soy sauce cakes was able to collect $10.2 \mathrm{mg} / 50 \mathrm{~g}$-feed of daidzein and $18.8 \mathrm{mg} / 50 \mathrm{~g}$-feed of genistein. The length of time the sample is retained in the mixer can be varied by changing the agitation speed, the number of baffle plates and the diameter of the mixer cylinder outlet. This solid-liquid extractor is therefore applicable in a wide range of food waste recycling processes.
\end{abstract}

\section{Introduction}

Food waste is an important new resource that includes a number of materials valuable for human health, such as flavonoids, polyphenols and capsaicins. In Japan, the total annual food waste is about 20 million $\mathrm{t}$, and the environmental load associated with this, as well as the social implications, is increasing. At the same time, a large amount of foodstuff, such as grain, corn, fish, and vegetables, is being imported into Japan from other countries. Development of a resource recycling society is therefore urgently needed (Hano et al., 2001; Hano and Sakoda, 2002).

Soy sauce, which is composed mainly of soybeans, wheat and salt, is a traditional and indispensable seasoning in Japan. The process of soy sauce manufacturing is shown in Figure 1. The steamed soybeans and wheat are initially roasted in a reaction chamber then crushed and fermented resulting in formation of soy sauce known as Koji. Brine solution, a mixture of water and salt, is then added to the Koji resulting in unrefined soy known as Moromi. After fermenting and aging the unrefined soy, the resultant product is divided into pure soy sauce and waste using a compressing machine. This waste is known as a soy sauce cake.

Received on December 27, 2005. Correspondence concerning this article should be addressed to H. Yamamoto (E-mail address: yhideki@kansai-u.ac.jp).
In Japan, about $100,000 \mathrm{t}$ of soy sauce cake is discharged each year, and although it has been used as a digestible protein, it is incinerated due to the declining livestock industry. A soy sauce cake contains about 5-10 wt\% salt; therefore, incineration results in corrosion of the furnace and environmental problems related to, for example, dioxin generation. However, amino and organic acids, and soybean isoflavone, as well as a number of other useful components remain in the soy sauce cake (Matsuda, 1998; Funazukuri et al., 2001; Nakashimada et al., 2002). So far, soybean isoflavone in the form of daidzein and genistein has been confirmed in soy sauce waste. Because soybean isoflavone is physiologically active, various protective effects on, for example, lifestyle diseases have been reported (Degenhard and Winterhalter, 2001; Yamori et al., 2001; Yang et al., 2001). The purpose of this research was to selectively separate isoflavone and salt from soy sauce cakes, and develop new separation equipment for practical use.

\section{Experimental Methods}

Extraction of flavonoids (daidzein and genistein) and salt from soy sauce cakes was carried out using various solvents: methanol, ethanol, 1-propanol, 2-propanol, acetone, $n$-hexane, water, and a combination of the above. Ten grams of soy sauce cake and $100 \mathrm{~cm}^{3}$ of solvent were mixed then shaken well in an Erlenmeyer 


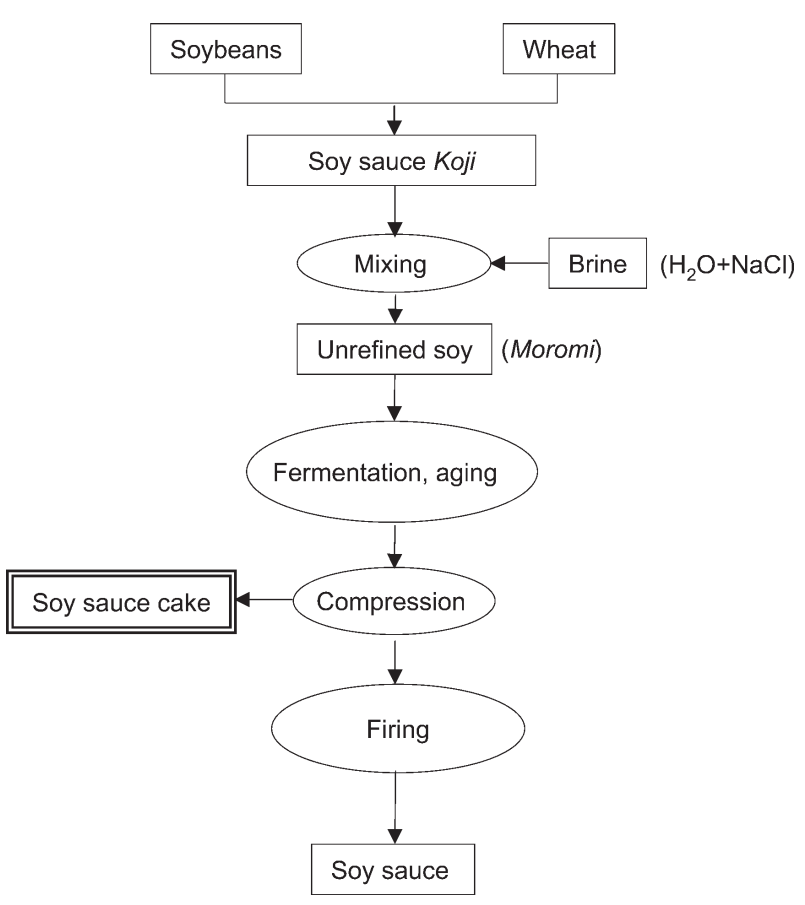

Fig. 1 Flow chart showing the manufacturing of soy sauce

flask at $318 \mathrm{~K}$ for $30 \mathrm{~min}$. After filtration of the cakesolvent mixture under reduced pressure, the amount of flavonoids dissolved in the solvent was measured using high performance liquid chromatography (HPLC; PU610-1X, GL Sciences, Inc.). Salt in the solvent was determined using a digital salinometer (ES-421, Atago Co.).

\subsection{Analysis of flavonoids extracted from soy sauce cake using HPLC}

HPLC was used for concentration measurements of daidzein and genistein extracted from soy sauce cakes. A chromatographer was equipped with a UV detector (UV620, GL Sciences, Inc.) and column (Inertsil ODS-3, GL Sciences, Inc.). The eluant used in this analysis was a mixture of methanol, THF, and phosphate buffer $(40 \mathrm{mmol}, \mathrm{pH} \mathrm{3.0})$ at a ratio of 30:20:50. HPLC was performed under the flow rate of $1.0 \mathrm{~mL} / \mathrm{min}$ and the column temperature of $40^{\circ} \mathrm{C}$. The $\mathrm{UV}$ detector was set at the wavelength of $254 \mathrm{~nm}$.

\section{Results and Discussion}

\subsection{Analysis of daidzein and genistein by LC-MS}

Table 1 shows the chemical structures and molecular weights of the main soybean isoflavones. A chromatogram of the condensate obtained by ethanol extraction using LC-MS is shown in Figure 2. Daidzein and genistein were detected by ionizing as 252.9 and 268.9 , confirming the existence of both compounds in the soy sauce cake used in this research.
Table 1 Chemical structures and molar weights of flavonoid compounds

Daidzein Genistein

\subsection{Extraction of isoflavone and salt using various solvents}

Figure 3(a) shows the amount of desalination resulting from extraction with various solvents. Desalination was highest with water $(590 \mathrm{mg} / 10 \mathrm{~g}-$ feed) and lowest with $n$-hexane $(0 \mathrm{mg} / 10 \mathrm{~g}$-feed). Figure 3(b) shows the amount of daidzein and genistein extracted with the same solvents. Acetone was shown to be the optimal extraction solvent for both compounds, resulting in 5.4 and $11.0 \mathrm{mg} / 10 \mathrm{~g}$-feed, respectively. Neither flavonoid was sufficiently extracted with water or $n$-hexane.

Overall, the extraction experiments revealed that daidzein, genistein and salt are barely extracted with $n$-hexane, although this solvent successfully extracted soy and other oils contained in the soy sauce cake. The amounts of extracted daidzein, genistein, and salt seemed to decrease with increasing hydrophobe strength.

\subsection{Extraction using a mixture of solvents}

Figures 4(a)-(d) show the amounts of flavonoids and salt extracted using various binary mixtures of extract solvent. The amount of flavonoids extracted increased while the amount of desalination decreased with mixed solvents containing water and alcohol. The amounts of flavonoids extracted using methanol + water, ethanol + water, and acetone + water were maximal as shown in Figures 4(a)-(c). The concentrations that gave maximum values were $50 \mathrm{~mol} \%$ methanol + water, $25 \mathrm{~mol} \%$ ethanol + water and $30 \mathrm{~mol} \%$ acetone + water, respectively.

\subsection{Development of a separation process for flavonoids and salt}

Judging from the fundamental extraction tests, ethanol and acetone are suitable for the extraction of soy flavonoids, while water, methanol, and ethanol are most appropriate for desalination. These results suggest that an alcohol or aqueous alcohol solution is suitable for extracting salt from soy sauce cakes. If an alcohol or aqueous alcohol solution is used in the separation process, solid-liquid separation becomes a simple operation because the density difference between the soy sauce cake and the extract solvent is increased. In addition, if the extract solvent doesn't include water, no wastewater will be produced during the recycling process. 


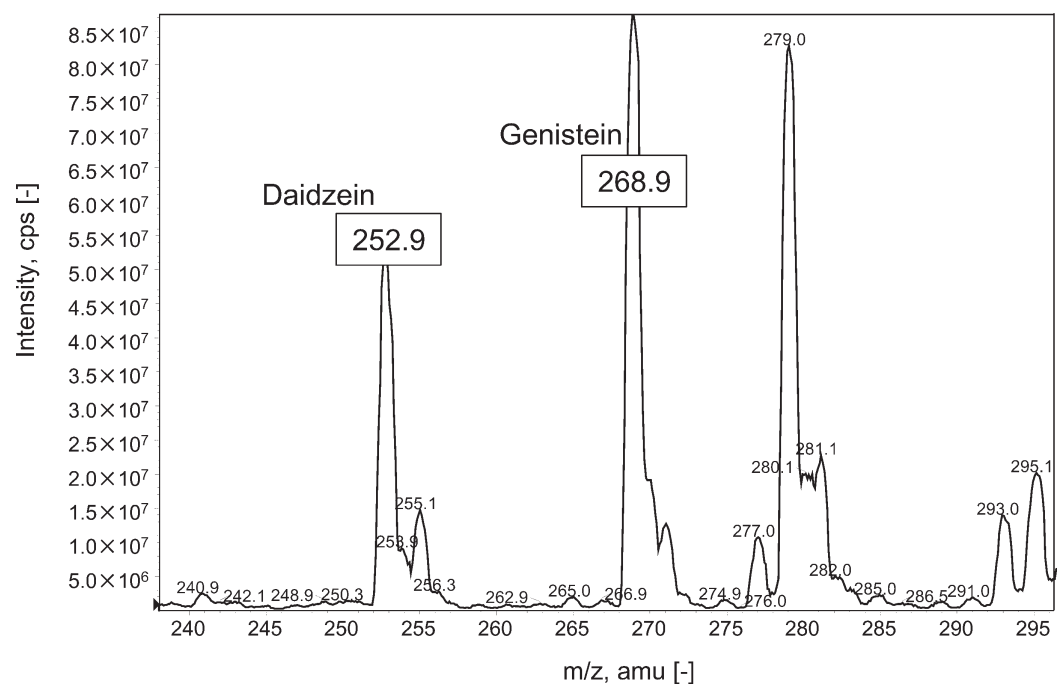

Fig. 2 Analysis of daidzein and genistein by LC-MS

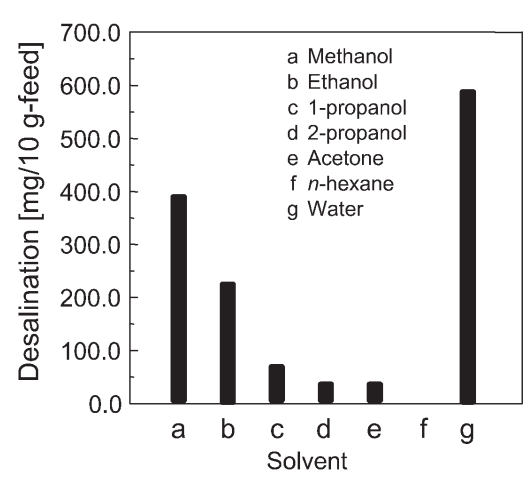

(a) Desalination

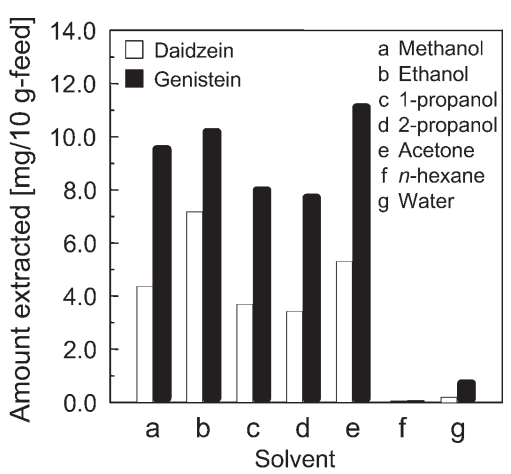

(b) Extracted flavonoids

Fig. 3 Extraction of salt and flavonoids using various solvents

The process of the separation of a soy sauce cake using acetone, and methanol or ethanol is described in Figure 5. After crushing, the soy sauce cake is introduced with acetone into a solid-liquid extractor for flavonoid extraction.

The extracted flavonoid solution is then condensed using a distillation process under reduced pressure and the distilled acetone is recycled. The soy sauce cake remaining in the extractor is removed and introduced once again for desalination with methanol or ethanol. As with the flavonoid solution, the extracted salt is condensed using a distillation process under reduced pressure and the collected methanol/ethanol is recycled.

\section{The Solid-Liquid Extractor}

\subsection{Dual cylinder solid-liquid extractor}

Figure 6(a) shows a schematic diagram of the dual cylinder solid-liquid extractor used to extract flavonoids and salt; a photograph of the extractor is shown in Figure 6(b). The extractor contains two cylinders, an internal cylinder with a capacity of $1.5 \mathrm{dm}^{3}$ as a mixer and an external cylinder with a capacity of $4.5 \mathrm{dm}^{3}$ as a settler. The soy sauce cake and extract solvent are stirred in the mixer cylinder from where the flavonoids and salt are then extracted, respectively. A residual soy sauce cake and extract solvent are discharged into the settler cylinder; the residual soy sauce cake is then precipitated as waste from the lower part of the cylinder and the extraction solvent is collected from the upper part as overflow. The residence time the soy sauce cake remains in the mixer can be controlled by the agitation speed, the number of baffle plates, and the diameter of the mixer cylinder outlet. The features of the dual cylinder solid-liquid extractor presented in this research make it applicable in a wide range of food waste recycling processes.

\subsection{Extraction performance of the dual cylinder solid-liquid extractor}

Extraction performance was examined using the presented mixer-settler extractor. Fifty grams of soy 


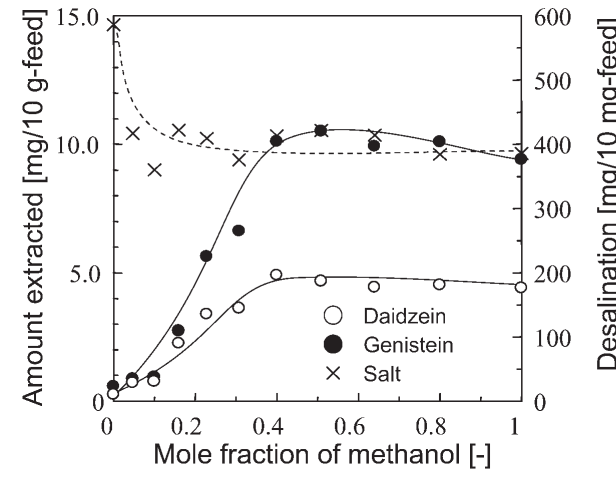

(a) Methanol-Water

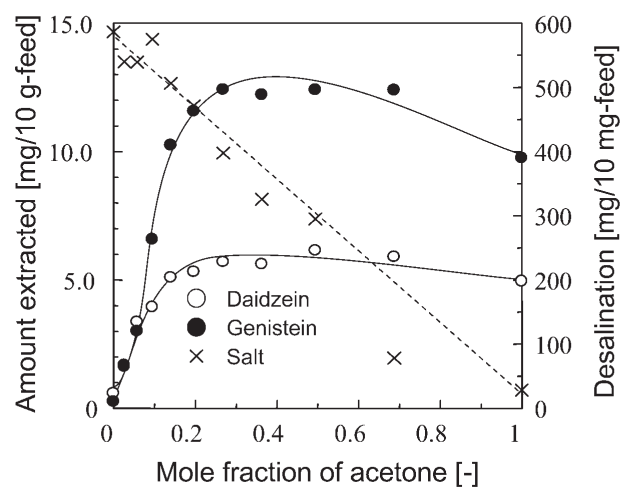

(c) Acetone-Water

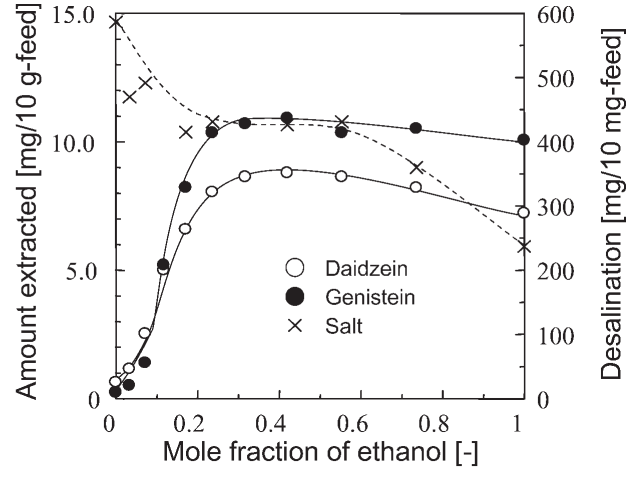

(b) Ethanol-Water

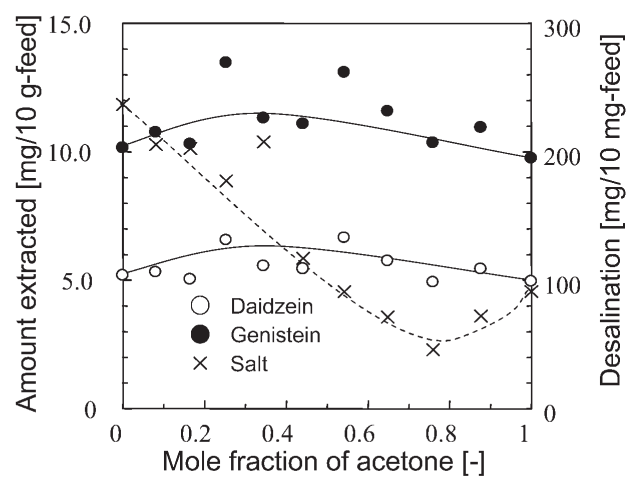

(a) Acetone-Ethanol

Fig. 4 Extraction of flavonoids and salt using binary solutions

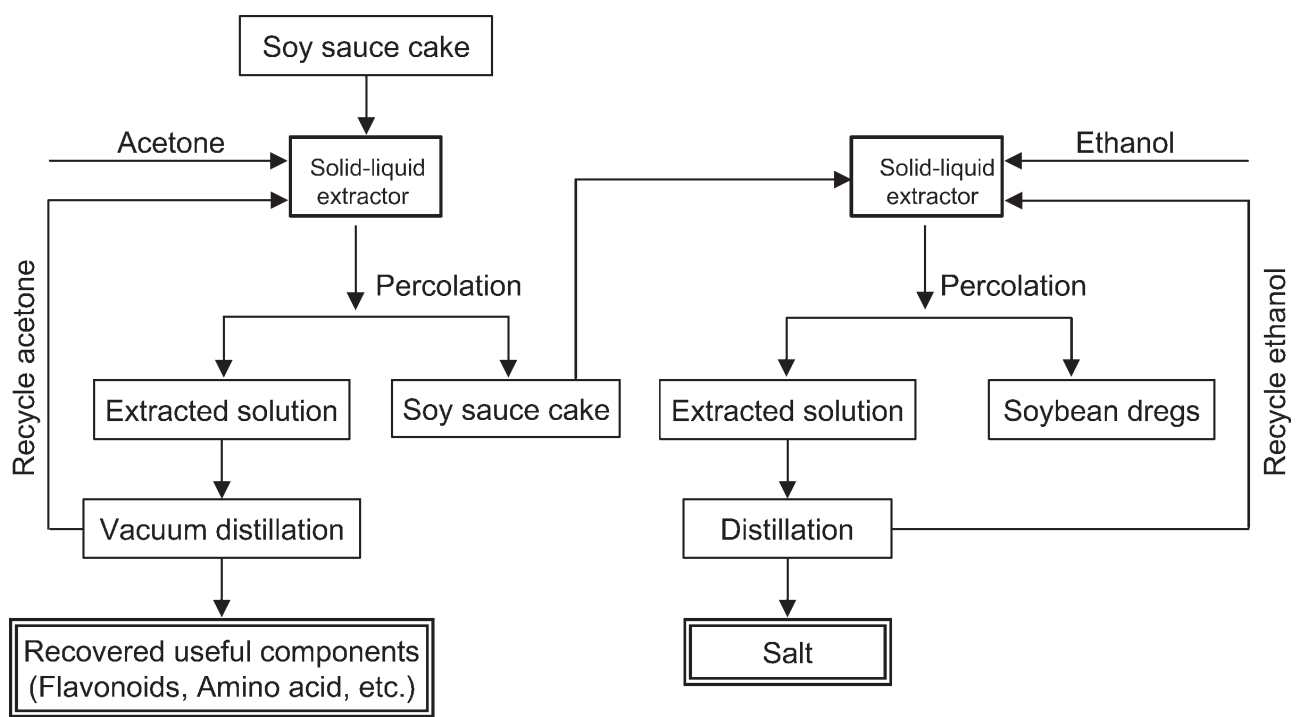

Fig. 5 Flow chart showing the separation of flavonoids and salt

sauce cake was introduced with a 50:50 (v/v) mixture of ethanol-water. The agitation speed and flow rate were maintained at $300 \mathrm{rpm}$ and $200 \mathrm{~cm}^{3} / \mathrm{min}$, respec- tively. The soy sauce cake and solvent were then stirred in the mixer and after $7 \mathrm{~min}$ all the residual solid had been discharged into the settler as a result of gravitation. 


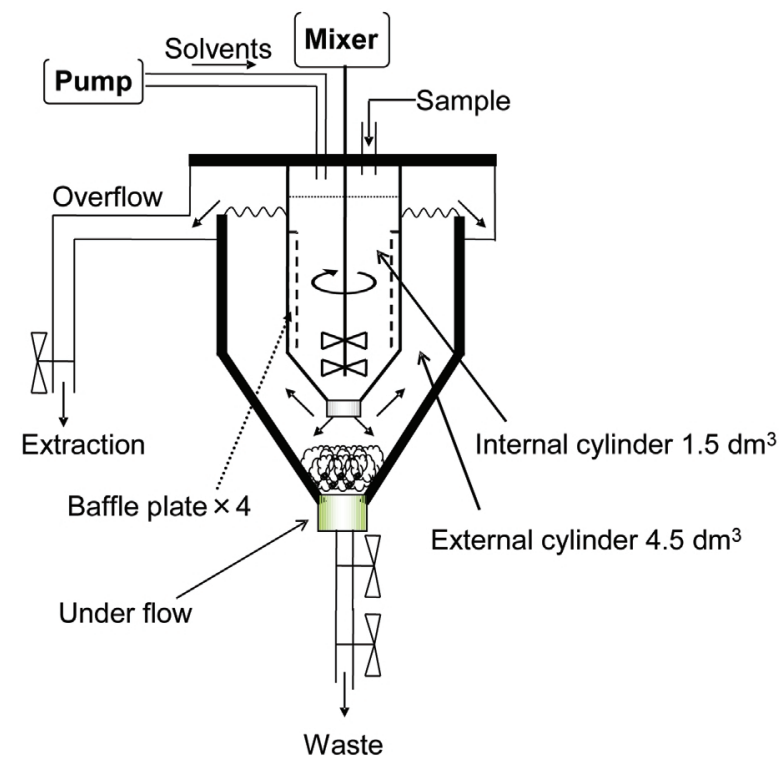

(a)

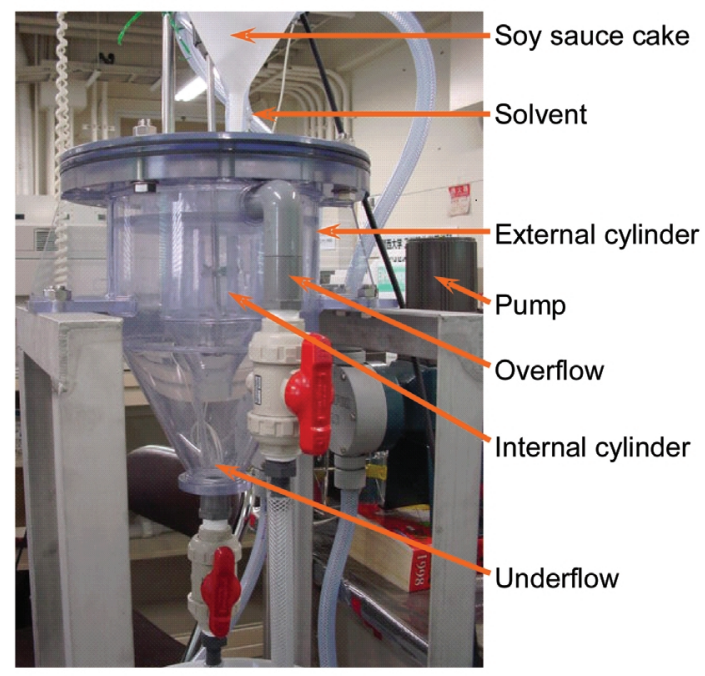

(b)

Fig. 6 Schematic diagram and photograph of a solid-liquid extractor

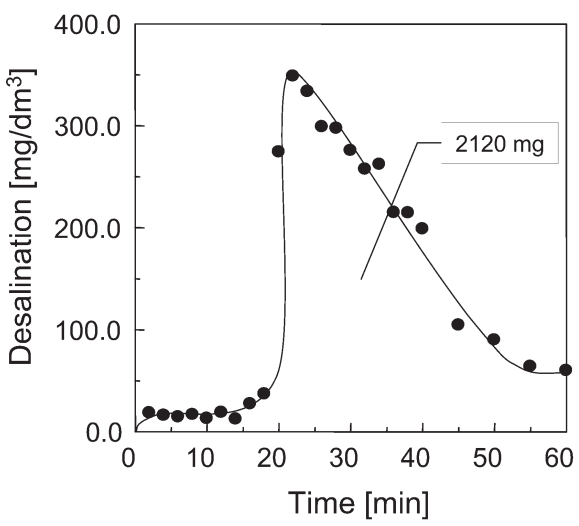

(a) Desalination

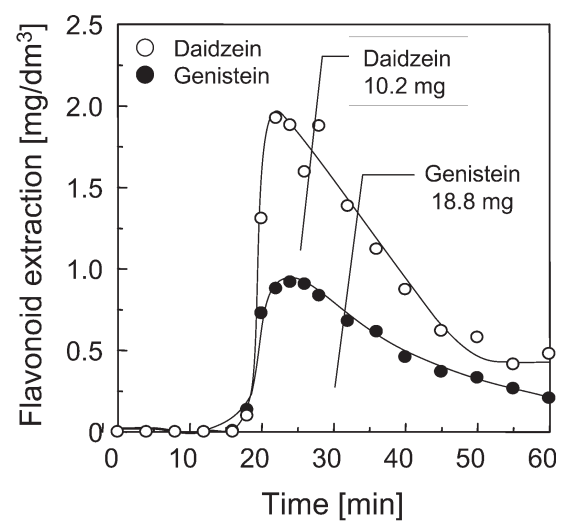

(b) Extracted flavonoids

Fig. 7 Time course of desalination and flavonoid extraction

Figures 7(a) and (b) show the time course of desalination and flavonoid extraction.

The amount of desalination was $2120 \mathrm{mg} / 50 \mathrm{~g}$ feed, and since $50 \mathrm{~g}$ soy sauce cake contains about 3000 $\mathrm{mg}$ of salt, the percentage of desalination was approximately $73.2 \%$. The amounts of daidzein and genistein extracted were 10.2 and $18.8 \mathrm{mg} / 50 \mathrm{~g}$-feed, respectively. The amounts extracted by butch operation using a 50:50 (v/v) mixture of ethanol-water are 25.4 and $53.7 \mathrm{mg} / 50 \mathrm{~g}$-feed, respectively; therefore, the percentage of extraction with the presented extractor was $40.4 \%$ for daidzein and $35.0 \%$ for genistein. Although the amount of flavonoids extracted here were lower than with butch operation, it is possible to improve this by optimizing the length of time the sample remains in the mixer.

\section{Conclusions}

In this study, it was possible to extract salt from soy sauce cakes not only with water but also with alcohols. The amount of desalination with water was $590 \mathrm{mg} / 10 \mathrm{~g}$-feed, while that with methanol was about $70 \%$ of this value. Desalination could not be performed successfully with acetone or $n$-hexane. The amounts of flavonoids in the form of daidzein and genistein extracted from $10 \mathrm{~g}$ of soy sauce cake were 3.4-7.2 and $7.2-11.0 \mathrm{mg}$, respectively, while extraction with water was minimal. Moreover, the amount of flavonoids extracted increased while the amount of desalination decreased with aqueous alcohol solutions.

The dual cylinder solid-liquid extractor presented here for separation of flavonoids and salt from a soy 
sauce cake was able to collect $10.2 \mathrm{mg} / 50 \mathrm{~g}$-feed of daidzein and $18.8 \mathrm{mg} / 50 \mathrm{~g}$-feed of genistein. The length of time the sample is retained in the mixer can be there varied by changing the agitation speed, the number of baffle plates, and the diameter of the mixer cylinder outlet. This solid-liquid extractor is applicable in a wide range of food waste recycling processes.

\section{Acknowledgment}

This research was supported by the Research and Development Organization of Industry-University Cooperation from the Ministry of Education, Culture, Sports, Science and Technology of Japan.

\section{Literature Cited}

Degenhardt, A. and P. Winterhalter; "Isolation and Purification of Isoflavones from Soy Flour by High-Speed Countercurrent Chromatography," Eur. Food Res. Technol., 213, 277-280 (2001)
Funadukuri, T., S. Uchida, N. Hirose and S. Kagei; "Conversion of Soy Sauce Cake by Steam Explosion Treatment," Environmental Science, 14, 359-365 (2001)

Hano, T. and K. Sakoda; "Zero-Emission Technologies in Food Industries" (in Japanese), Chemical Engineering, 66, 261-266 (2002)

Hano, T., M. Hirata, H. Takanashi and K. Nakamura; "Mass Flow Analysis of Soy-Sauce Production Process and Dry Distillation of Lees," Environmental Science, 14, 325-331 (2001)

Matsuda, S.; "Study on Antioxidative Substances of Soy Sauce Lee" (in Japanese), Journal of Brewing Society of Japan, 93, 263269 (1998)

Nakashimada, Y., M. Kobayashi and N. Nomura; "Treatment of the Waste from the Process of Soy Sauce Brewing and Its Useful Application," Bio Industry, 60, 807-812 (2002)

Yamori, Y., S. Ota and S. Watanabe; Soybean Isoflavones, pp. 3947, Saiwaishobo, Tokyo, Japan (2001)

Yang, F., Y. Ma and Y. Ito; "Separation and Purification of Isoflavones from a Crude Soybean Extract by High-Speed Counter-Current Chromatography," J. Chromatogr. A, 928, 163-170 (2001) 